\title{
LA PACIFICACIÓN DE LA GUAJIRA POR EL INGENIERO ANTONIO DE ARÉVALO. SOBRE EL PROYECTO DE DEFENSA DE SABANA DEL VALLE
}

\author{
THE PACIFICATION OF THE GUAJIRA BY ENGINEER \\ ANTONIO DE AREVALO. ON DEFENSE PROJECT \\ SABANA DEL VALLE
}

\author{
Manuel Gámez Casado \\ mangamcas1@alum.us.es
}

\begin{abstract}
El presente artículo tiene como principal fin aportar nuevos datos sobre una de las expediciones menos estudiadas de las realizadas en el área caribeña por el ingeniero Antonio de Arévalo, la centrada en la pacificación de los indios de La Guajira. Para ello tuvo que recurrir tanto a sus habilidades diplomáticas, como a sus conocimientos militares. A tal fin proyectó una serie de fuertes destinados a proteger las zonas estratégicas. Entre todas sus propuestas se ha prestado especial atención a su proyecto para la población costera de Sabana del Valle, en la actual frontera entre Colombia y Venezuela.

Palabras claves: Caribe, Nueva Granada, ingeniero militar, fortificaciones, Agustín Crame.
\end{abstract}

The following article intend to provide new material about the pacification of the Indians of La Guajira by Antonio de Arevalo as it was one of the least studied expeditions that were made to the Caribbean area by the engineer. Thus He had to resort to his diplomatic skills and his military knowledge . For this porpuse He designed a number of forts to protect strategic areas. Among all his proposals "the project for the coastal town of Sabana del Valle", in the border between Colombia and Venezuela.

Keywords: Caribbean, New Granada, military engineer, fortifications, Agustín Crame.

Dentro de las diferentes expediciones que el ingeniero Antonio de Arévalo realizó en las provincias del Caribe sur, con el fin de pacificar las poblaciones indígenas de la zona, se encuentra la realizada a La Guajira, que dio lugar a distintos informes geográficos, religiosos y sociales. Esta tarea se vio reflejada en una amplia memoria que contenía la información necesaria para solucionar dicho problema y que incluía leyendas locales, aspectos de la religión, descripciones 
y relatos recogidos "in situ" por Arévalo entre los guajiros ${ }^{1}$. Dicha expedición se inició en 1774, en plena construcción de muchas de las fortificaciones de Cartagena de Indias en las que estaba ocupado, lo que denota una importante capacidad de trabajo por parte del ingeniero ${ }^{2}$. En esa misión a tierras que hoy son fronterizas entre Colombia y Venezuela, Arévalo tuvo ocasión de elaborar diferentes proyectos de fortificaciones que permitirían la defensa de los enclaves más importantes. La localización de una serie de noticias sobre estos planes y propuestas, permiten aportar nuevos datos sobre la expedición llevada a cabo por el ingeniero español y su trascendencia.

Con anterioridad a la llegada de Arévalo a La Guajira, se realizaron algunas incursiones en la zona, ya bajo el reinado de Carlos III. La proclamación de dicho monarca, así como la firma del Tercer Pacto de Familia, propició una importante alianza con Francia, así como la más absoluta enemistad con Inglaterra. Ello provocó que el imperio británico iniciase una serie de viajes por la costa del Caribe sur, alcanzando importantes contactos con los indios nativos, con los que mantenían contrabando y a quienes surtían de armamento y munición. Esto empeoraba la situación de las poblaciones sublevadas ante la Corona española, como era el caso de La Guajira. Así, desde los primeros años del reinado de Carlos III, con el fin de consolidar el gobierno español en la zona, el militar don Bernardo Ruiz protagonizó una importante comisión con el fin de pacificar dicha provincia. Una de sus primeras medidas fue la fundación de la ciudad de San Carlos, en la cual reunió a varios poblados indios de las poblaciones de Chimare y Bahía Honda. Sin embargo, la villa fue abandonada por parte de los indios pasado un año de su fundación, denotando una falta de control por parte de las autoridades españolas sobre la población indígena.

Para acabar con la falta de autoridad española, en 1773 se proclamó virrey a don Manuel de Guirior, quien encargó al entonces coronel Antonio de Arévalo la reducción de los indios de La Guajira, así como su definitiva pacificación. A partir de aquí, Arévalo embarcó en Cartagena de Indias el 20 de noviembre, para llegar a

${ }^{1}$ OLIVEROS DE CASTRO, María Teresa: "La Guajira y las ordenaciones de don Antonio de Arévalo", Revista de Historia Militar, 22, 1967, pp. 79-111.

${ }^{2}$ El ingeniero Antonio de Arévalo compaginaba sus labores edilicias en la consolidación del sistema defensivo de Cartagena de Indias con misiones de pacificación y relaciones diplomáticas con los indios de las provincias del Caribe sur. Así, anteriormente a la expedición que llevó a cabo en La Guajira, Arévalo había alcanzado acuerdos de pacificación con los indios de El Darién, como ya estudió ZAPATERO, Juan Manuel: "Expediciones españolas al Darién. La del ingeniero don Antonio de Arévalo en 1761", Revista historia militar, $\mathrm{n}^{\circ}$ 19, Madrid, 1965, pp. 50-51. Sobre la producción ingenieril de Antonio de Arévalo en Cartagena de Indias, véase SEGOVIA SALAS, Rodolfo: Las fortificaciones de Cartagena de Indias. Bogotá: 1992; ZAPATERO, Juan Manuel: Historia de las fortificaciones de Cartagena de Indias. Bogotá. 1979; y del mismo autor, El ingeniero militar de Cartagena de Indias don Antonio de Arévalo. 1742-1800. Sevilla, 1981. 
Rio Hacha seis días después. Las primeras actividades practicadas en la zona fueron de reconocimiento, ya que el ingeniero procuró conocer el estado de la tropa, la artillería disponible, así como el sistema defensivo utilizado en la plaza. En un primer momento, no aceptó llevar a cabo una campaña de represión absoluta, pues confiaba en atraer de forma pacífica a los guajiros. Para conseguir este fin, el ingeniero pretendía acomodar el modo de vida de las tribus, no teniendo que deambular por el territorio en busca de víveres dado el carácter nómada de este pueblo. Por ello, consideró como uno de los principales métodos para asentarlos el de fijar nuevas fundaciones en zonas que determinasen la mejora de sus relaciones con los españoles, debiendo ser un terreno fértil, con abundancia de agua, pasto y leña. Además, distinguió entre dos tipos de fundaciones. Las destinadas a españoles por un lado, y las utilizadas por los indios, obedeciendo a un plan de ordenación social que pretendía la pacificación definitiva del territorio ${ }^{3}$.

Así, ciudades como Pedraza pasaron a ser controladas íntegramente por los españoles, al igual que Bahía Honda, quien adoptó el sobrenombre de San José, eligiendo estas plazas por estar bien situadas, en un terreno seco pero con agua de buena calidad. La nueva ciudad de San José de Bahía Honda, se equipó rápidamente con una batería para defender su costa, lo que denota que la organización de la vida en estas nuevas ciudades requirió de una preparación importante, ya que se trataba de acomodar de la mejor forma a los diferentes pobladores. Para ello, la elección de un número proporcionado de habitantes era crucial, lo que ha permitido la pervivencia hasta la actualidad de estas nuevas fundaciones ideadas por Antonio de Arévalo.

Ejemplo de la españolización que sufrieron las ciudades, fue la construcción de una iglesia y una plaza mayor en el municipio de Orino, copiando los modelos urbanísticos que la Corona había implantado en América. Durante todo el periodo virreinal, la copia de los modelos artísticos peninsulares sirvió como método de unificación de un territorio de grandes dimensiones, por lo que esta constante se continuó utilizando en la pacificación guajira. Una vez conseguidos estos planteamientos, Antonio de Arévalo se dispuso a regresar a Rio Hacha con el resto de la tropa. Sin embargo, la noche anterior a la salida de la expedición, los indios se llevaron nueve caballos del comandante, teniendo que hacerles frente el capitán de artillería don José Galluzo junto a 50 milicianos ${ }^{4}$. Los indios huyeron, siendo perseguidos por los soldados españoles, los cuales pedían de forma pacífica la entrega de los animales. Finalmente, los nativos se retiraron

${ }^{3}$ El primer informe realizado por Antonio de Arévalo donde describió la situación de la zona fue publicado por POLO ACUÑA, José: "Antonio de Arévalo y la frontera de la península de la Guajira. 1770-1776", Taller de Historia, Cartagena. 2001, pp. 213-250.

${ }^{4}$ El estudio más completo sobre el capitán José Galluzo fue realizado por SARMIENTO PÉREZ, José: José Galluzo y Páez. Capitán general y primer presidente de la Junta Suprema de Extremadura. 1746-1817. Badajoz, 2011. 
entregando los caballos, lo que muestra un cierto temor por parte de éstos ante las tropas hispanas.

Después de esta operación, y tras asentar definitivamente la ciudad de Bahía Honda, solo quedaba someter a los indios cozinas, los más violentos de la zona. Sobre la procedencia étnica de este grupo se ha discutido en la historiografía. No obstante, se ha aceptado su incorporación a la población guajira, al hablar el mismo idioma y practicar las mismas costumbres, a pesar de vivir al margen de la sociedad. Este aislamiento, lo ratificó Arévalo, quien los describía como "enemigos comunes a todos los de la provincia porque roban a todos los que pueden". La pacificación de este pueblo no se consolidó, si bien, la capitulación obtenida por el comandante José Galluzo permitió un mayor control del mismo ${ }^{5}$.

Los diferentes expedientes que Arévalo redactó para informar al virrey de la situación de las ciudades guajiras, constituyen un corpus de documentos fundamentales para conocer las condiciones sociales que se daban en cada una de ellas. Este contacto era necesario para suplir la posible falta de víveres, armamento o soldados en las diferentes plazas, a fin de lograr una correcta defensa de la zona. El hallazgo de una serie de cartas del ingeniero prueba esta relación constante con el virrey. Así, en la fechada el 17 de marzo de 1776, escrita en Rio Hacha por Antonio de Arévalo y dirigida al virrey don Manuel Antonio de Flores, se especifica la necesidad de enviar a Santa Marta, cargados en una balandra, a cuarenta hombres con fusiles para reemplazar a los muertos y heridos, así como una artillería con soldados y 10.000 pesos para los gastos que pudiesen surgir. No obstante, Arévalo era consciente de que sería perjudicial para la estrategia defensiva el acopiar a las tropas, al no haber suficientes víveres para mantenerlas, por lo que el envío de soldados debía ser escalonado. Por otra parte, el ingeniero se refiere a la provincia de Maracaibo, insistiendo en la necesidad de establecer una relación formal con el gobernador para que se facilitasen los auxilios necesarios, enviándose en embarcaciones que impidiesen la comunicación de los contrabandistas con los indios de Apiesi. Además, solicitó al virrey que ordenase al gobernador que diera 1.000 pesos al que entregase, vivo o muerto, al autor de las revueltas acaecidas en la zona. Pero si éstas continuaban se debían asegurar los puertos de mayor importancia, como Bahía Honda o Maracaibo, para posteriormente ocuparse de la reducción de los indios con los refuerzos llegados desde Cartagena ${ }^{6}$. Ello demuestra la complejidad estratégica que supuso la correcta defensa de La Guajira, lo que significó un constante trasiego de mercancías y armamento con el fin de controlar las revueltas.

5 Ibídem, pp. 105-109.

${ }^{6}$ Archivo General de Indias (en adelante AGI). Santa Fe, 1242, "Carta Antonio de Arévalo". Rico hacha, 17 de marzo de 1776. Además, puede consultarse KUETHE, Allan: "The pacification campaign on the Rio Hacha frontier, 1772-1779", The Hispanic American Historical Review. Vol. 50, n 3, 1970, pp. 467-481. 
Las órdenes del virrey eran claras, castigar de forma tajante a los indios de Apiesi y restablecer el orden en la ciudad, la cual había sido enormemente castigada desde el inicio de las oleadas revolucionarias. Uno de los principales inconvenientes que se presentaban para la pacificación de estos indios era la falta de agua y provisiones en dicha plaza. De esta forma, se enviaron unas milicias con destino a Bahía Honda, las cuales debían ir por el camino más corto, con el fin de evitar un atentado. Aquí, se cargarían los burros y caballos con víveres y munición, llevando además vacas para poder comer su carne. De allí irían por tierra hasta Apiesi, mientras que dos balandras reforzarían la expedición por mar. Este grupo salió el día 12 de abril, contando con 325 hombres destinados para asegurar definitivamente la pacificación. Además, el día 10 de dicho mes, el gobernador se comprometió a visitar la zona, con la intención de que su presencia atemorizara a los indios ${ }^{7}$.

De esta forma, el virrey don Manuel Antonio Flores, en respuesta a toda la información recibida por Arévalo, confirmó su conocimiento de la grave situación de Apiesi, así como del conjunto de la provincia de Rio Hacha, en una carta escrita el 30 de mayo de $1776^{8}$. En ella reconoce haber leído el diario en el que José Galluzo, gobernador de la provincia, describía lo ocurrido en la zona. En dicho escrito, se confirma que la expedición estaba formada por 505 hombres, muchos de ellos pertenecientes al regimiento fijo de Cartagena, mientras que otros eran nativos voluntarios pertenecientes a Santa Marta, Maracaibo o Sabana del Valle, incorporados a cambio de librarse de los pagos de los tributos durante un año ${ }^{9}$. Ante esto, resultaba evidente que los indios conocían mejor el terreno, por lo que en dicho viaje se intentó retener una posible incorporación de los Pusiarines y Macuyra, ya que si lograban unirse a los de Apiesi, la resistencia podía ser muy dura ${ }^{10}$. Otro de los objetivos que se perseguía en esta campaña de pacificación, era el de detener al indio Vichuana, líder de las campañas revolucionarias, al que debían entregar para practicarle un castigo en presencia de otros nativos, con el fin de atemorizarles ${ }^{11}$. Por su parte, los indios habían detenido a algunos españoles a fin de canjearlos. Por ello, Arévalo añadió en su diario de la expedición

\footnotetext{
7 AGI. Santa Fe, 1242, "Carta 7 Abril 1776".

${ }^{8}$ AGI. Santa Fe, 1242, "El virrey de Santa Fe da cuenta del estado en que se halla la expedición de la provincia de Rio de Hacha".

${ }^{9}$ Los pueblos nativos debían pagar 4 pesos por año, de forma que si en dicha expedición participaron 100 hombres, se les perdonó finalmente 400 pesos.

${ }_{10}$ Sobre los diferentes pueblos de indios de la zona sur del Caribe, véase AA.VV.: Castas, mujeres y sociedad en la Independencia. Bogotá, 2010.

11 Vichuana protagonizó uno de los más sangrientos levantamientos el 30 de enero de 1776 en el pueblo de La Cruz, organizando una rebelión contra el gobierno de Nicolás Díaz de Perea. SUÁREZ ROLDÁN, Juan Camilo: "Lectura de una simulación histórica poética de un extravío", Coherencia, nº 2, Vol. 2, 2005, p. 59.
} 
la necesidad de obtener víveres y dinero para llevar a cabo tal intercambio, solicitando ayuda tanto al gobernador de Santa Marta como al de Maracaibo ${ }^{12}$.

Hasta que no se consiguiesen tales propósitos, el virrey insistió en la necesaria actuación de los misioneros para solucionar la falta de víveres. No obstante, los religiosos tenían que refugiarse continuamente para huir del peligro que les amenazaba, ante los continuos levantamientos de las ciudades. En ellas, se robaban las iglesias y se les amenazaba, por lo que se solicitaba la rápida actuación de dicha expedición a fin de que cumpliese todos estos objetivos. Por último, el virrey, de acuerdo a la solicitud de Antonio de Arévalo, aprobó la creación de fuertes guarnecidos, necesitando para ello cañones de poco tamaño, fusiles, espadas y municiones, solicitando el rápido envío de este armamento ante la importante crisis que se planteaba. A partir de aquí, el enfrentamiento con los indios de Apiesi resultó ser uno de los más cruentos de los llevados a cabo en la zona, lo que no permitió una consolidación del dominio español en el territorio.

La importante crisis generada en 1776 debido a la violencia de las revoluciones protagonizadas por los indios, generó una importante documentación mediante la cual, Antonio de Arévalo intentaba facilitar la defensa de la zona. Partiendo de esta consideración, si nos atenemos al mapa realizado por el ingeniero con fecha de 15 de octubre de 1776, podemos ver en él las diferentes ciudades que sufrían las revueltas ${ }^{13}$. En el mapa se muestra toda la provincia de Rio Hacha, desde Santa Marta a Maracaibo. En la leyenda que la acompaña se explica que lo representado eran las ciudades ocupadas por los indios guajiros, así como las de nueva fundación española, hechas a fin de conseguir la pacificación general de la zona, según el proyecto de Arévalo. En el mapa, las zonas de Apiesi y Maracaibo adquieren un importante papel, en tanto en cuanto, eran las de mayor repercusión en las oleadas revolucionarias. Así, en esta representación se aprecia que el ingeniero quiso mostrar una división entre las ciudades dedicadas a los españoles y las de ocupación indígena.

Una vez que se asentaron las ciudades destinadas a la vivienda de los españoles, y siguiendo el plan establecido por Arévalo, se procedió a fundar poblados para los indios. Este fue el caso de poblaciones como Carrizal, Chimare, Macuira o Sabana del Valle, siendo este último un enclave estratégico en la configuración geográfica de los indios. Por ello, Arévalo tuvo especial celo en la protección de estas localidades, de forma que una vez fundadas, procuró que en ellas no se estableciese un comercio ilegal de armas, así como el contrabando de otros productos,

${ }^{12}$ A ambos gobernadores se les solicitó la cantidad de 5.000 pesos, a fin de que pagase la deuda de 3.394 impuesta por los indios como rescate de las tropas, utilizándose el resto del dinero para satisfacer las necesidades de la expedición que se dirigía a Apiesi.

13 AGI, MP-Panamá, 190BIS. mapa general de la provincia de Hacha situada entre la de Santa Marta y Maracaibo, para la inteligencia de su extensión y límites". Santa Fe, 15 de octubre, 1776. Figura 1. 
ya que, como se ha dicho, estas eran las razones principales por la que los indios adquirieron poder frente a las tropas españolas. El estudio de los diferentes mecanismos de defensa para el aislamiento de estas nuevas localidades habitadas por indios, era hasta ahora inédito pues se han podido documentar diferentes proyectos que Arévalo llevó a cabo en algunos de estos enclaves.

Una de las primeras noticias conocidas para proceder a la defensa de la plaza de Sabana del Valle, en la costa occidental de Maracaibo, se recoge en la carta que escribió Arévalo dirigida al teniente coronel de ingeniero en segunda de los ejecitos don José Díaz de Pedregal, fechada el 14 de abril de $1777^{14}$. En ella, explicó que con fecha del 31 de marzo de 1776, el secretario de cámara del virrey, don Francisco Iturrate, le remitió la Real Orden del 10 de septiembre de 1775 mediante la cual el monarca aceptó la realización de un fuerte en la costa occidental del golfo de Maracaibo. El costo de la obra lo asumiría la Compañía Guipuzcoana, siendo auxiliado para la realización del proyecto por el gobierno de la provincia, quien debía verificar cualquier operación ${ }^{15}$. En dicha carta, Arévalo hizo referencia a la imposibilidad de que Agustín Crame llevase a cabo la obra, debido a que sus trabajos se estaban desarrollando lejos de La Guajira, de forma que se le encomendaba a Pedregal la realización de un plano que explicase la construcción de dicho fuerte ${ }^{16}$. En él debía hacer referencia al presupuesto necesario para tal empresa.

En la carta, Arévalo añadió la necesidad de defender el fondeadero de Sabana del Valle, no solo contra los indios de la zona, sino también contra los ingleses que proveían de armas y munición a los guajiros. De esta forma, el ingeniero tuvo muy presente la necesidad de fortificar la plaza para evitar así el comercio de armas, principal razón por la que los indios ofrecieron una férrea resistencia al ejército español. Además, se concertó que dicho fuerte estuviese adaptado a las condiciones topográficas del terreno, utilizando las ventajas y desventajas del mismo para mejorar su eficacia defensiva. Arévalo recomendaba que en la ejecución del proyecto, se utilizasen materiales seguros, además de situar de una forma

${ }^{14}$ Sobre la producción del ingeniero José Díaz de Pedregal, véase AA. VV.: Los ingenieros militares en España, siglo XVIII: repertorio biográfico e inventario de su labor científica y espacial. Barcelona, 1983, pp. 149-150.

${ }^{15}$ La Real Compañía Guipuzcoana de Caracas fue una de las sociedades más activas en el comercio español en el Caribe sur. Su actividad y repercusión económica en la zona fue estudiada por CIERBIDE MARTINENA, Ricardo: "La Compañía Guipuzcoana de Caracas y los vascos en Venezuela durante el siglo XVIII", Revista internacional de los estudios vascos, Vol. 42, n 1, 1997, pp. 63-75.

${ }^{16}$ Agustín Crame había trabajado en la defensa de Maracaibo, realizando la reforma y ampliación del castillo de San Carlos y el de Zaparas. No obstante, en torno a 1774, el ingeniero se encontraba reforzando la defensa del fuerte de San Juan de Ulúa frente a Veracruz, lo que hacía imposible que se hiciese cargo de dicho proyecto en el Caribe sur. AA.VV.: Los ingenieros militares en España..., op. cit., p. 131. 
correcta cada una de las partes del conjunto. Para ello, se examinaron las avenidas, quebradas, anegadizos y aguas que hubiese en la zona para que el fuerte pudiese adaptarse con corrección. La necesidad de conocer el terreno se basaba en saber si existían materiales de buena calidad y a qué distancia se encontrarían, ya que según la constitución del edificio, así sería su costo. Por último, Arévalo excusó la imposibilidad del capitán e ingeniero don Juan Jiménez Donoso para que llevase a cabo el proyecto, ya que se encontraba enfermo de gravedad. Así, se pensó en Pedregal para que encabezase la comisión, emplazándole a una reunión futura en la cual dicho ingeniero junto a Arévalo pudiesen discutir el plano y el proyecto del fuerte ${ }^{17}$.

A partir de aquí, la relación epistolar entre Arévalo y Díaz de Pedregal fue una constante, ya que se debía trazar un nuevo sistema de fortificación que defendiese un enclave tan problemático para la Corona desde las primeras fundaciones del siglo XVI. En una carta enviada del primero al segundo, fechada el 15 de abril de 1777, dejó constancia que la defensa de dicho enclave era fundamental para evitar el contrabando ilícito con extranjeros, quienes proveían de armas y municiones a los indios. De esta forma, se quiso zanjar el modo de abastecimiento de pertrechos y armas, ya que si se terminaba con las relaciones que los guajiros mantenían con los ingleses, se debilitarían sus fuerzas y por ende se facilitaba la defensa de la zona ante ataques indígenas. Para llevar a cabo tal propósito, Arévalo dio una serie de instrucciones a Pedregal, que tenían como base fundamental la construcción de un fuerte en la zona. Según cuenta Arévalo, junto a esta carta remitió un mapa que permitiría un mejor entendimiento de la zona, siendo éste copia del que había levantado en fecha próxima el gobernador de Maracaibo don Domingo Armario.

Por otro lado, Arévalo insistió en la necesidad de reconocer el puerto y el terreno antes de proceder a la realización del proyecto final. Así, una vez llegados a Sabana del Valle, se debía examinar con precisión, tanto su agua, como sus playas y costas, siendo necesario para establecer las salidas y entradas. A partir de aquí, se podía levantar el plano, teniendo en cuenta que el puerto debía satisfacer toda la demanda de la costa, utilizando balizas y líneas en el dibujo que marcarían la entrada hasta el surgidero. El plano, hasta ahora inédito, nos permite conocer el proyecto con total precisión ${ }^{18}$. No obstante, mediante la descripción que ofrece Arévalo, podemos saber que se proyectaron dos baterías de 30 varas (25 metros) de largo cada una, dispuestas en ángulo de forma que sus fuegos se cruzasen, para así establecer un sistema defensivo infalible que permitiese la autodefensa de las

17 AGI. Santa Fe, 1242, “Carta de Antonio de Arévalo a José Díaz Pedregal”. Cartagena de Indias. 14 de abril, 1777.

${ }^{18}$ Instituto de Historia y Cultura Militar. Cartoteca Archivo General Militar de Madrid. "Plano del fuerte para el puerto de Sabana del Valle". Antonio de Arévalo. 1777. Figura 2. 
mismas, como ya se hizo en el canal de Bocachica, principal entrada a la bahía de Cartagena de Indias ${ }^{19}$. Estas baterías estaban protegidas por dos baluartes, unidos por una amplia cortina con la que se pretendía cerrar una de las avenidas reconocidas como fundamentales para el tránsito del comercio ilegal. Tanto los dos baluartes como la cortina intermedia fue concebida por Arévalo como unos paramentos de mampostería sencilla, atronerada, con 3 varas (2,5 metros) de alto en la zona interior y 3,5 (2,9 metros) en la zona exterior, facilitando así el trabajo de los fusileros. Con ello, el fuerte proyectado contaría con una capacidad de 4 cañones por cada una de las baterías, así como 20 pedreros y 50 hombres de guarnición. Además, se contaba desde este primer momento con la participación de los propios vecinos en caso de guerra, los cuales podían refugiarse en el edificio, denotando la importancia que este proyecto tendría.

Las baterías proyectadas debían ser bajas y a barbeta, para que sus fuegos provocasen el mayor daño posible al enemigo. Así, se permitía que atacasen tanto de frente como desde algún ángulo, lo que les hacía totalmente eficaces para la destrucción del enemigo. Arévalo concibió que si no se conseguía batir al enemigo desde uno de los lados, los cañones podían pasarse al costado contrario a través de explanadas, si bien, la construcción de éstas no debía aumentar considerablemente el costo del proyecto. Estas explanadas debían realizarse con hormigón, con la anchura necesaria para el correcto paso tanto de las tropas, y de la artillería. Junto a ello, para mejorar la defensa de las baterías, propuso la realización de una barrera de piedra, que impidiese que las embarcaciones se acercasen a la fortificación. Este elemento se situaría al pie de la batería, cercano a su paramento exterior, con una medida de 3 varas (2,5 metros) de alto, elevándose la muralla sobre este elemento 5 pies y medio (1,8 metros), por lo que dichas baterías contarían con una altura total de 4,3 metros, señalándose en el plano con la letra T.

Por otro lado, en previsión de que el terreno donde se debía construir el fuerte estuviese poco elevado sobre el nivel del mar, Arévalo encomendó que se hiciesen las diferentes explanadas que comunicarían distintos puntos del edificio por la parte interior, mediante una serie de rampas que se debían adaptar en inclinación y altura a los requisitos del lugar. Ello se explica con detenimiento en el plano, solucionando dicha problemática. Esta zona interior del fuerte debía estar ocupada por una serie de edificios destinados al cuerpo de guardia, como son la capilla, el cuartel, los almacenes para víveres y municiones, así como habitaciones para el comandante, un oficial, capellán, cirujano y guardias. El edificio contaría con un aljibe que solucionaría los problemas de abastecimiento de agua, ya que solo se habían encontrado pozos en una zona cercana a la recién fundada ciudad de

${ }^{19}$ En dicha plaza, se dispusieron las baterías de San Fernando y San José, las cuales cruzaban su fuego impidiendo el paso del enemigo, lo que además permitía defender un mayor espacio de terreno a lo largo del canal. MARCO DORTA, Enrique: Cartagena de Indias. La ciudad y sus monumentos. Sevilla, 1951, p. 288. 
Santa Ana. También, el hecho de colocar estos depósitos de agua cerca de los baluartes, permitiría conducir a ellos el agua necesaria, teniendo una capacidad de almacenaje suficiente como para suplir un año de falta de lluvias. En el plano estas dependencias aparecen detalladas y sombreadas con suma precisión.

Una vez determinadas las características morfológicas de la fortificación, se debía determinar la artillería que se debía utilizar en ella. Al respecto, era necesario tener en cuenta que en el puerto de Sabana del Valle no podían entrar fragatas debido a que la profundidad máxima de la zona era de 2,5 brazas ( 4,5 metros), pudiendo acceder a dicha zona solo las balandras. Así, Arévalo aconsejó en el informe que tratamos, la utilización de la artillería del calibre 16, para hacer frente a posibles incursiones no solo de balandras, sino de barcos que pudiesen cargar un armamento de mayor calibre.

Por último, una vez que Antonio de Arévalo dio las instrucciones necesarias para la correcta disposición de cada uno de los elementos que conformarían el fuerte, se debería reconocer el terreno de las inmediaciones con el fin de saber en qué parte se podrían obtener los materiales para su ejecución. Además, era necesario tener en cuenta la construcción de habitaciones provisionales que debían construirse en la parte exterior del fuerte, con el fin de ser utilizadas para los artesanos que trabajasen en la obra, con los cuales era necesario contactar para conocer el coste definitivo. Una vez llevado a cabo todo este proceso, el teniente coronel don José Díaz Pedregal debía pasar a Maracaibo para entregarle al gobernador el plano del fuerte, su costo y demás documentación, con el fin de conseguir la aprobación del virrey. Arévalo insistió en que si en Maracaibo se encontrase con el brigadier Agustín Crame, se reuniesen para tratar de la construcción del fuerte, al ser uno de los más reputados ingenieros españoles en el Caribe.

A partir de aquí, una vez entregados los documentos en la capital de la región y tras contactar con los artesanos que ejecutarían el fuerte, Díaz de Pedregal remitió el 8 de noviembre de 1777, una relación con el costo que podría suponer para el virreinato su realización con el fin de que fuese aprobado tanto por Arévalo como por el gobierno. El hecho de querer proteger la costa occidental de la bahía de Maracaibo, hizo que la Corona ratificase las primeras medidas para la construcción de dicho fuerte, según las instrucciones anteriormente analizadas. El presupuesto definitivo fue desarrollado por Antonio de Arévalo, como ingeniero director de los reales ejércitos, y Díaz de Pedregal, el cual actuaba como director adjunto en la construcción de este fuerte. Conjuntamente, dicha fortificación contaría con un comandante, dos oficiales, un destacamento de cincuenta hombres, un capellán, un guarda para los almacenes, un cirujano y un vivandero, así como con ocho cañones de calibre 16 o 24. Dicho personal ya había sido requerido por Arévalo en su informe.

Para plantear el presupuesto definitivo del fuerte, hubo que tener en cuenta que el único material cercano era la piedra de la costa, por lo que el uso de otros materiales que disminuirían el coste de la obra, como la madera, la cal o el ladrillo, quedaba 
descartado. Sí se aceptó la construcción de los dos pozos cercanos a la ciudad de Santa Ana, los cuales se situaban a dos millas $(3,2 \mathrm{~km})$ del terreno a fortificar. Junto a todas estas circunstancias, las fuertes mareas que interrumpían en ocasiones la navegación por la zona del saco de Maracaibo, se sumaba al conjunto de incidencias que se tuvieron en cuenta para el cálculo definitivo del presupuesto. Así, el costo de la albañilería fue el primero de los asuntos a tratar. Aquí se incluyó la realización de los cimientos y fosos de la obra, teniendo que extraer un total de 2.755 varas cúbicas (2.309 metros cúbicos) de tierra, así como la excavación de 528 varas cúbicas (442 metros cúbicos) para la realización de los aljibes que se situarían dentro de los baluartes. También la fabricación de 1.336 varas cúbicas (1119 metros cúbicos) de terraplenes que se debían colocar en las baterías y la construcción de los paramentos de mampostería ordinaría que ascendían a 4.403 varas cúbicas (3690 metros cúbicos). Se detalló dentro del presupuesto la necesidad de contar con 225 varas (188 metros) de hormigón para la realización de las obras. La suma de todos estos trabajos se estimó en 20.704, 5 pesos. Otro de los apartados del presupuesto es el referente a la carpintería, en el cual se incluyeron todo el conjunto de vigas necesarias para la realización de balaustres, destacando las 92 varas de madera que se utilizarían para las armaduras de los tejados. También contenía la madera necesaria para fabricar las habitaciones exteriores donde residirían los artesanos que construían el fuerte. La suma total de los trabajos de carpintería ascendió a 21.851,5 pesos.

Tras ello, se desglosa el precio de una serie de trabajos y materiales necesarios para la realización de todo el equipamiento del fuerte. Se presupuestan desde las alcayatas hasta las herramientas necesarias para la fabricación, así como la compra de seis burros para la conducción del agua. Igualmente uno de los gastos necesarios, debido a la situación geográfica de la fortificación, era el referente a la compra de tres balandras, cuya misión era la de transportar los materiales necesarios. Dos de ellas se utilizarían para la conducción, mientras que la tercera actuaría como retén en el fondeadero. En suma, se estimaba un presupuesto total para la construcción del fuerte y su equipamiento de $34.866,1$ pesos $\mathrm{y} 3 / 4$ reales.

Este informe presupuestario se concluye con la advertencia de que la construcción debía hacerse a la vista de los indios enemigos, por lo que convenía realizar una trinchera donde colocar unos ciento cincuenta guardias que defendiesen la obra, con sus correspondientes pertrechos y municiones. La intención era que los operarios no perdiesen tiempo en su trabajo. Por ello mismo, se hizo necesaria la realización de unas estancias destinadas al descanso de los artesanos, así como al almacenaje de sus víveres, a fin de protegerse de las borrascas y los vientos. Además, en el caso de llevar a cabo todas las construcciones interiores que Arévalo había ideado con el fin de equipar la fortificación, el costo de la misma ascendería en $44.978,7$ pesos $^{20}$.

${ }^{20}$ AGI. Santa Fe, 1242, "Presupuesto fuerte. José Diaz Predegal". Maracaibo, 4 de agosto de 1777. 
Sin embargo, los esfuerzos de Arévalo por defender la zona mediante esa fortificación fueron en balde, ya que finalmente se optó por las soluciones que Agustín Crame proponía. Su informe preveía la reforma y ampliación del castillo de San Carlos y el de Zaparas, obras iniciadas en 1780 por el ingeniero Casimiro Isava de Oliver. Estos fuertes se encargaban desde el siglo XVI de la defensa de Maracaibo, por lo que tras la aceptación de las propuestas de Crame se decidió centrar todos los esfuerzos económicos en estas empresas y olvidar las propuestas de Arévalo ${ }^{21}$.

Con todo ello, se pone de manifiesto la importante labor desarrollada por el ingeniero Antonio de Arévalo no solo en el diseño de fortificaciones y defensas, sino también en el ejercicio de relaciones diplomáticas con las diferentes tribus del Caribe sur que, alentadas y apoyadas por las tropas británicas, se habían sublevado contra el poder español. Sus propuestas para solventar los problemas con los indígenas demuestran un alto conocimiento de la realidad geográfica, física y étnica, valores esperables en una persona con una formación tan esmerada como la de un ingeniero militar. La investigación aquí desarrollada aporta novedades relevantes sobre la actividad de un importante ingeniero de la monarquía hispana, al que siempre se había vinculado con los problemas defensivos de Cartagena de Indias, pero del que hay que valorar otras actuaciones tanto en el área del Caribe sur, como en la zona media de dicho mar.

Fecha de recepción: 30 de septiembre de 2015.

Fecha de aceptación: 20 de noviembre de 2015.

${ }^{21}$ GUTIERREZ, Ramón: Fortificaciones en Iberoamérica. Madrid, 2005, pp. 233-234. 


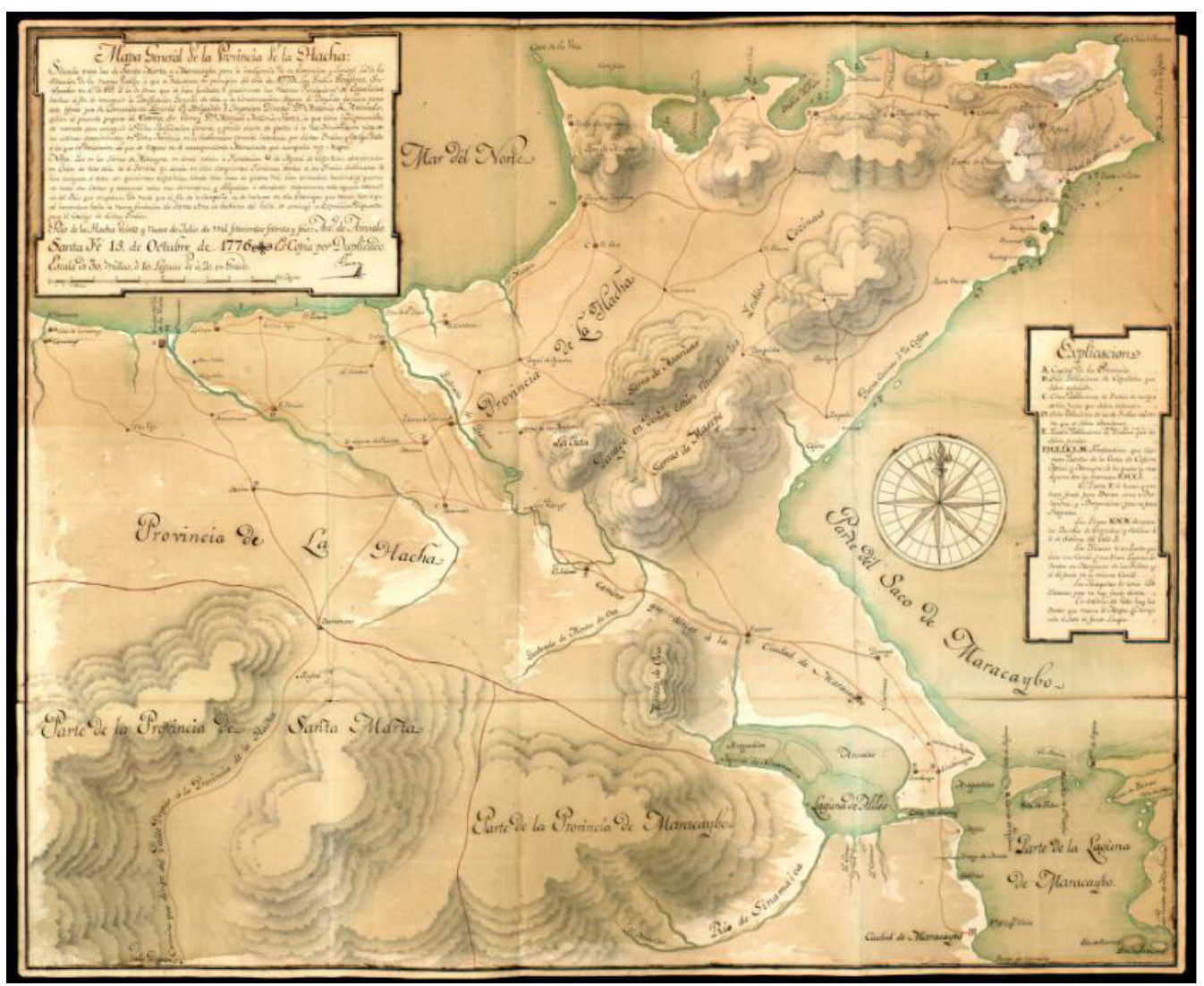

Figura 1. Plano de la península de La Guajira realizado por Antonio de Arévalo en 1776. Ministerio de Educación, Cultura y Deporte. Archivo General de Indias. MPPanamá,190BIS. 


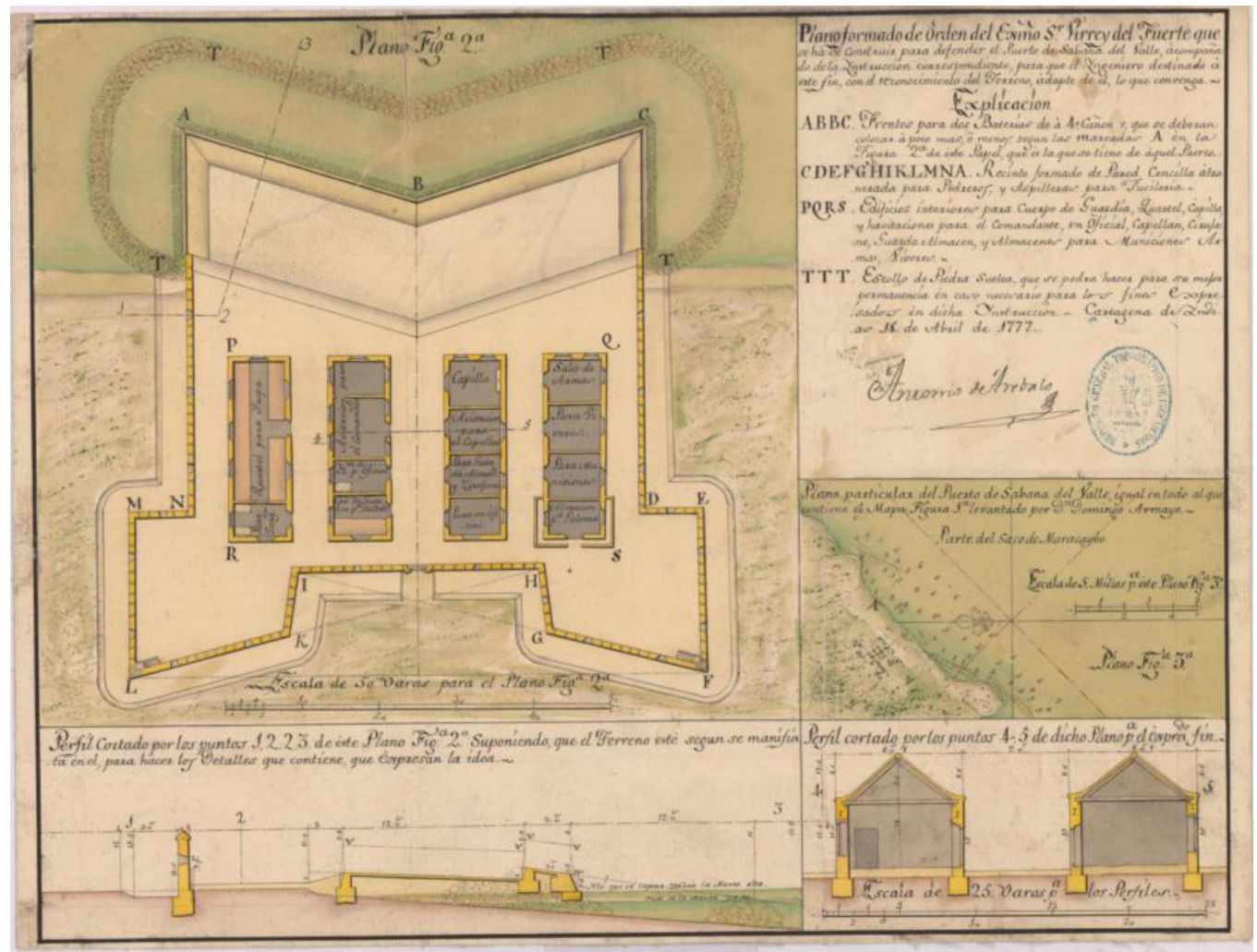

Figura 2. Plano del fuerte para el puerto de Sabana del Valle. Antonio de Arévalo. 1777. Ministerio de Defensa. Instituto de Historia y Cultura Militar. Cartoteca Archivo General Militar de Madrid. Sign: COL- 8/2. 